This study aimed to evaluate serum level of sCXCL16 in patients with juvenile SLE (jSLE) for possible value of its estimation in assessing the disease activity and the extent of the disease.

Methods Twenty seven patients with jSLE and twenty healthy controls were included. Demographic and clinical features of the patients were recorded. Disease activity was assesssed using the SLE Disease Activity Index (SLEDAI). The serum levels of sCXCL16 were determined by Eliza and correlated with clinical and laboratory parameters and with SLEDAI. Renal biopsy was done to all patients.

Results The serum levels of sCXCL16 in jSLE patients were higher than controls $(\mathrm{p}<0.001)$, they were also siginificantly higher in patients with alopecia or malar rash than other jSLE . Positive correlation was identified between serum levels of sCXCL16 and SLEDAI score. There was a significant positive correlation between sCXCL16 levels and severity of lupus nephritis as assessed by renal biopsy. Serum levels of sCXCL16 were positively significantly correlated with the 24 hour urine protein, ANA, SBP, DBP AND ESR 1st hour. Serum sCXCL16 level was significanly negatively correlated with C3 serum level.

Conclusions Soluble CXCL16 could become a useful serological marker of disease activity and severity of renal involvement in jSLE patients.

\section{AN UNUSUAL CASE OF PAEDIATRIC LUPUS WITH ISOLATED RETINAL INVOLVEMENT}

${ }^{1}$ A Jindal ${ }^{*},{ }^{2} V$ Gupta, ${ }^{1} S$ Suri, ${ }^{1}$ A Rawat, ${ }^{1}$ A Gupta, ${ }^{1} S$ Singh. ${ }^{1} P G M E R$, Paediatrics, chandigarh, India; ${ }^{2} P G M E R$, Ophthalmology, chandigarh, India

\subsection{6/lupus-2017-000215.134}

Background and Aims Eye involvement in SLE is seen in around one third of patients. Retinal involvement is an uncommon but potentially vision threatening complication.

Methods To report the case of a young boy with isolated retinal involvement as the only manifestation of lupus.

Results A 9 year old previously healthy boy presented with complaints of fever, vomiting, seizures and alteration of sensorium. He had signs of raised intracranial pressure and rest of the examination was unremarkable (Investigations in Table 1 and 2). He was managed as a case of viral meningo-encepahlitis though his CSF examination and neuroimaging revealed no abnormality. He was incidentally detected to have cotton wool
Abstract 134 Table 2

\begin{tabular}{|l|l|}
\hline Investigations & Result \\
\hline CSF & \\
Microscopy & No cells seen \\
Sugar (mg/dl)/protein (mg/dl) & $102 / 91$ \\
Gram stain and AFB stain & Negative \\
TB PCR & Negative \\
Culture & Sterile \\
HSV DNA PCR & Negative \\
\hline IgM Mycoplasma serology & Negative \\
\hline IgM Leptospira serology & Negative \\
\hline IgM Rickettsia serology & Negative \\
\hline IgM dengue serology & Negative \\
\hline Serum widal & Negative \\
\hline Peripheral smear for malaria antigen & Negative \\
\hline HIV EuSA & Negative \\
\hline IgM EBV-VCA & negative \\
\hline Mantoux & 0 mm \\
\hline Chest X-ray & Normal \\
\hline Gastric lavage for AFB stain (3 samples) & negative \\
\hline Urine microscopy (3 times) & No RBCs, protein- nil \\
\hline ANA (done twice, IIF using Hep2 cells) & $4+$ speckled \\
\hline C3(mg/dL) & 183 (normal range: 50- \\
\hline C4 (mg/dL) & 150 ) \\
\hline Anti ds DNA antibodies & 35 (normal range: 20-50) \\
\hline Immunoblot for extractable nuclear & Negative \\
\hline antigen (ENA) & negative \\
\hline Direct Coombs test & \\
\hline Skin biopsy for lupus band & Negative \\
\hline Antiphospholipid antibodies & (both anti IgG and anti \\
\hline & C3d) \\
\hline
\end{tabular}

spots in both eyes. After recovering from acute illness, he was also noted to have low vision in both eyes (light perception at 3 feet) Subsequently,a fundus fluorescein angiography was

Abstract 134 Table 1 Laboratory investigations

\begin{tabular}{|c|c|c|c|c|c|c|}
\hline Investigations & Day 1 & Day 3 & Day 5 & Day 7 & Day 10 & Day 20 \\
\hline Haemoglobin (gm/L) & 91 & 91 & 105 & 81 & 72 & 76 \\
\hline WBC counts $\left(\times 10^{9}\right.$ cells $\left./ \mu L\right)$ & 3.7 & 3.8 & 6.6 & 4.2 & 5.1 & 8.6 \\
\hline Differential counts (P/L/M/E) & $63 / 27 / 9 / 1$ & $73 / 26 / 6 / 1$ & $78 / 16 / 4 / 2$ & $63 / 29 / 8 / 0$ & $78 / 16 / 4 / 2$ & $68 / 30 / 1 / 1$ \\
\hline $\begin{array}{l}\text { Absolute lymphocyte counts (x } \\
10^{9} \text { cells/ } / L \text { L) }\end{array}$ & 1.0 & 1.0 & 1.0 & 1.2 & 0.8 & 2.6 \\
\hline Platelet counts $\left(\times 10^{9} / \mu \mathrm{L}\right)$ & 169 & 148 & 108 & 73 & 107 & 570 \\
\hline AST/ALT (U/L) & $53 / 46$ & $138 / 61$ & $195 / 65$ & $147 / 49$ & $146 / 56$ & $57 / 44$ \\
\hline Urea (mg/dl) & 34 & 18 & 34 & 44 & 19 & 25 \\
\hline Creatinine (mg/dL) & 0.7 & 0.7 & 1.2 & 0.9 & 0.6 & 0.4 \\
\hline
\end{tabular}




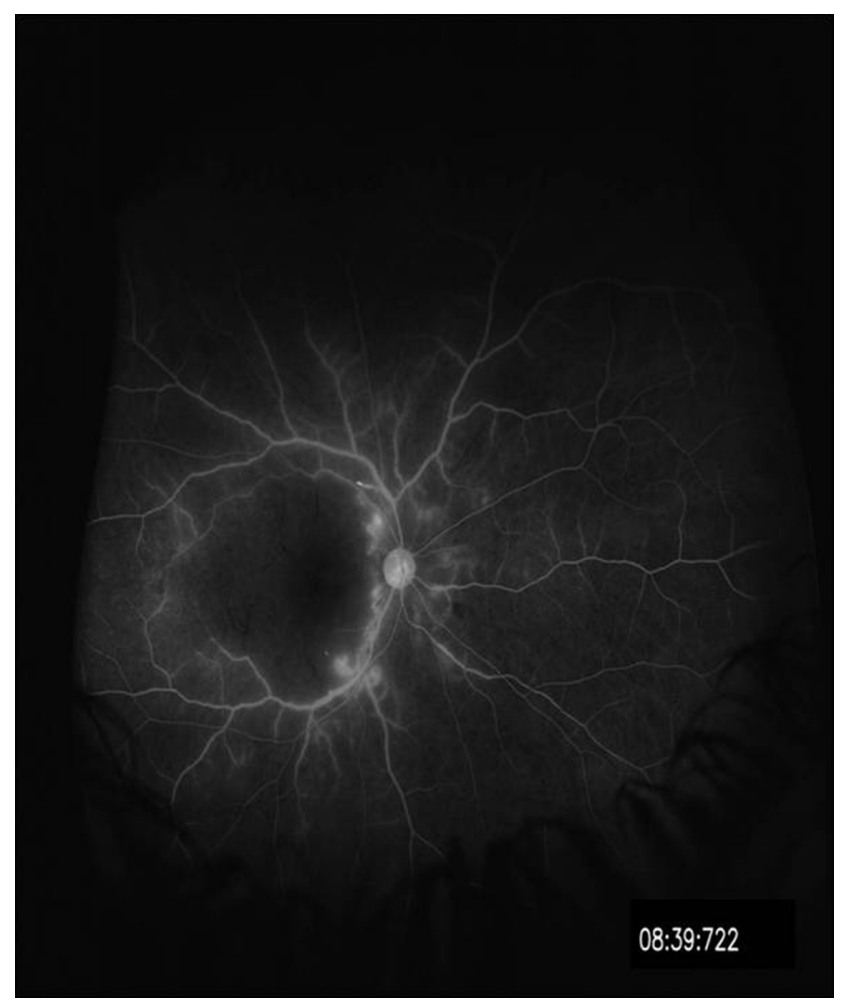

Abstract 134 Figure 1

done that revealed capillary 'drop-out', vessel wall staining and leakage of dye. (Figure 1) He had antinuclear antibodies (4+ speckled pattern). A diagnosis of SLE with isolated retinal involvement (vasculopathy) was considered. In view of severely impaired visual acuity, he was managed with injection cyclophosphamide (followed by maintenance mycophenolate mofetil) and pulse methylprednisolone (followed by oral prednsiolone). One year on follow up, his visual acuity has improved to $6 / 60$ in both eyes. Repeat FFA revealed significant decrease in the non perfused areas of retina.

Conclusions Eye involvement in paediatric lupus is underecognized entity and may need aggressive therapy.

\section{SKIN PEELING AND IRRITABILITY IN A YOUNG BOY WITH SYSTEMIC LUPUS ERYTHEMATOSUS- IS THERE AN OVERLAP WITH KAWASAKI DISEASE?}

A Jindal*, D Suri, A Gupta, A Rawat, S Singh. PGIMER, Paediatrics, chandigarh, India

\subsection{6/lupus-2017-000215.135}

Background and Aims Juvenile systemic lupus erythematosus (SLE) and Kawasaki disease can have several overlapping clinical and laboratory manifestations. But the co-occurrence of both disorders is extremely uncommon.

Methods To report the case of a young boy who had features of both SLE and KD

Results A 5 year old boy presented with fever for 2 months associated with photosensitive malar rash, oral ulcers and myalgias.On examination, he was irritable, had malar rash, a generalised erythematosus macular rash, oral ulcers, red and vertically cracked lips, redness of bulbar conjunctiva, cervical lymphadenopathy and hepatomegaly. Laboratory investigations are summarised in Table 1. All infectious disease workup was normal. He had hypocomplementemia, positive ANA, anti dsDNA and anti-nucleosome antibody. He was diagnosed as SLE and initiated on oral prednisolone. Fever subsided, transaminitis and leucopenia showed gradual recovery. One week later developed periungual skin peeling and he remained irritable. Laboratory investigations showed anaemia, thrombocytosis, persistently elevated ESR and high CRP (Table 1) A diagnosis of incomplete $\mathrm{KD}$ was proffered. $\mathrm{He}$ was given intravenous immunoglobulin (IVIG) (2 gm/ $\mathrm{kg})$, which led to prompt improvement in his irritability. He was initiated on aspirin $(3 \mathrm{mg} / \mathrm{kg} / \mathrm{day})$ and continued on oral prednisolone. Aspirin was discontinued 6 weeks later after confirmation of normal coronary findings on echocardiography. Beau's lines were noticed in the finger nails at this time. At 3 months followup, he continues to remain well. Serum transaminases, platelet counts, ESR and CRP have normalised. (Table 1)

Conclusions Overlap of KD and SLE is extremely uncommon. (Table 2)

Abstract 135 Table 1 Laboratory investigations

\begin{tabular}{|l|l|l|l|l|l|l|l|}
\hline $\begin{array}{l}\text { Investigation } \\
\text { Day of } \\
\text { Admission }\end{array}$ & $\begin{array}{l}\text { Hemoglobin } \\
(\mathrm{gm} / \mathrm{L})\end{array}$ & $\begin{array}{l}\text { White blood cell } \\
\text { count }\left(\times 10^{9} \text { cells } / \mathrm{L}\right)\end{array}$ & $\begin{array}{l}\text { Differential counts } \\
(\mathrm{P} / \mathrm{L} / \mathrm{M} / \mathrm{E})\end{array}$ & $\begin{array}{l}\text { Platelet counts } \\
\left(\times 10^{9} / \mathrm{L}\right)\end{array}$ & $\begin{array}{l}\text { ESR }(\mathrm{mm} \\
\text { in I" hr) }\end{array}$ & $\begin{array}{l}\text { CRP } \\
(\mathrm{mg} / \mathrm{L})\end{array}$ & $\begin{array}{l}\text { AST/ ALT } \\
(\mathrm{U} / \mathrm{L})\end{array}$ \\
\hline Day I & 76 & 3.3 & $30 / 60 / 8 / 2$ & 292 & 52 & 82 & $182 / 73$ \\
\hline Day 5 & 70 & 4.1 & $21 / 69 / 9 / 1$ & 269 & 83 & - & $816 / 287$ \\
\hline Day 9 & 76 & 7.3 & $54 / 38 / 6 / 2$ & 225 & - & - & $456 / 286$ \\
\hline Day 21* & 83 & 14.9 & $45 / 35 / 9$ & 510 & 61 & 16 & $88 / 139$ \\
\hline Day 60 & 100 & 13.6 & $49 / 41 / 9 / 1$ & 400 & 40 & 4 & $36 / 45$ \\
\hline
\end{tabular}

*IVIg was administered on day 21 